\title{
GRUPOS DE MACROINVERTEBRADOS DEL BENTOS DEL LAGO DE SANABRIA Y SU RELACION CON LA GRANULOMETRIA DEL SEDIMENTO
}

M. Rieradevall, C. Ibañez, I. Muñoz, M. Real \& N. Prat

Dept. Ecologia, Univ. Barcelona, Diagonal, 645; 08028 BARCELONA.

Palabras clave: sedimentos, granulometría, bentos, quironómidos, oligoquetos.

\section{ABSTRACT}

This study is designed to provide the first data on the structure and density of the macrobenthic communities from sublittoral and profundal areas of Lake Sanabria (NW Spain). This is an oligotrophic, cold monomictic and the largest natural lake in Spain. Three replicate Ekman grabs were collected at 10 sampling stations from sublittoral ( 7 $\mathrm{m})$ to the deepest parts $(46 \mathrm{~m})$, seasonally during 1990-91. Granulometric analysis of the sediments revealed a very fine silty sediment in the deepest parts of the lake from 11 to $50 \mathrm{~m}$ depth and the presence of some sand, and a more coarse composition in the sublittoral zone (less than $11 \mathrm{~m}$ depth). The sediment is poor in carbonates because the granitic lake watershed and rich in silicates and organic matter from allochthonous origin. The community is mainly composed of Oligochaeta and Chironomidae (mainly Micropsectra contracta). Densities range from a few individuals up to $6000 \mathrm{ind} / \mathrm{m}^{2}$, Oligochaeta being more abundant in the deepest parts and species richness higher in the sublittoral zones.

\section{INTRODUCCION}

El bentos de los lagos y embalses españoles empieza a ser conocido después de los estudios recientes del grupo de bentos del Departamento de Ecología de la Universidad de Barcelona y algunos datos procedentes de estudios de consultoras (Prat, 1976; 1978; 1979a, b; 1980a, b, c; Martínez-Ansemil \& Prat, 1984; Limnos, 1989; Real \& Prat, 1991; Prat \& et al., 1991; Real et al., 1992). Del lago de Sanabria existian sólo unos datos fraccionarios (Prat, 1980b). Con los trabajos recientes sobre el bentos del lago de Banyoles (Rieradevall, 1991; Rieradevall \& Prat, 1989; 1991; Prat \& Rieradevall, en prensa) faltaba por determinar con más detalle las características de los sedimentos y el bentos del lago de Sanabria que por ser el más grande de España y por su situación geográfica parecía interesante conocer. Así, junto con los datos de los embalses (Prat, 1980b; Real \& Prat, 1991; Real et al., 1992) se podría tener una visión global de nuestras comunidades bentónicas en los ecosistemas leníticos (Prat, 1992, este volumen).

\section{MATERIAL Y METODOS}

Las muestras se tomaron en cuatro ocasiones entre Junio de 1990 y Junio de 1991 en 10 estaciones de muestreo situadas a diferente profundidad en zonas distintas del lago cubriendo sus dos cubetas principales (Fig. 1). En cada ocasión y para cada estación se tomaron tres réplicas con una draga Ekman de $225 \mathrm{~cm}^{2}$ de área de

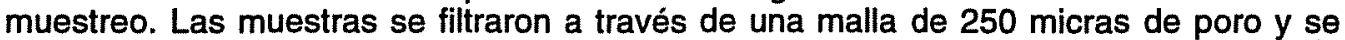
separaron "in vivo" en un laboratorio en la orilla del lago durante las 24 horas siguientes al muestreo. La separación se realizó por flotación con azúcar, siguiendo el método de Anderson (1959). En una ocasión se recogió de una de las dragas de cada punto de muestreo un core de $3 \mathrm{~cm}$ de diámetro para determinar la granulometría. Esta se analizó con el método clásico de la pipeta de Robinson. En una estación se hicieron, directamente de la draga, cortes cada $2 \mathrm{~cm}$ para observar la variación de la granulometría a lo largo del sedimento que se tomaba con la draga. 


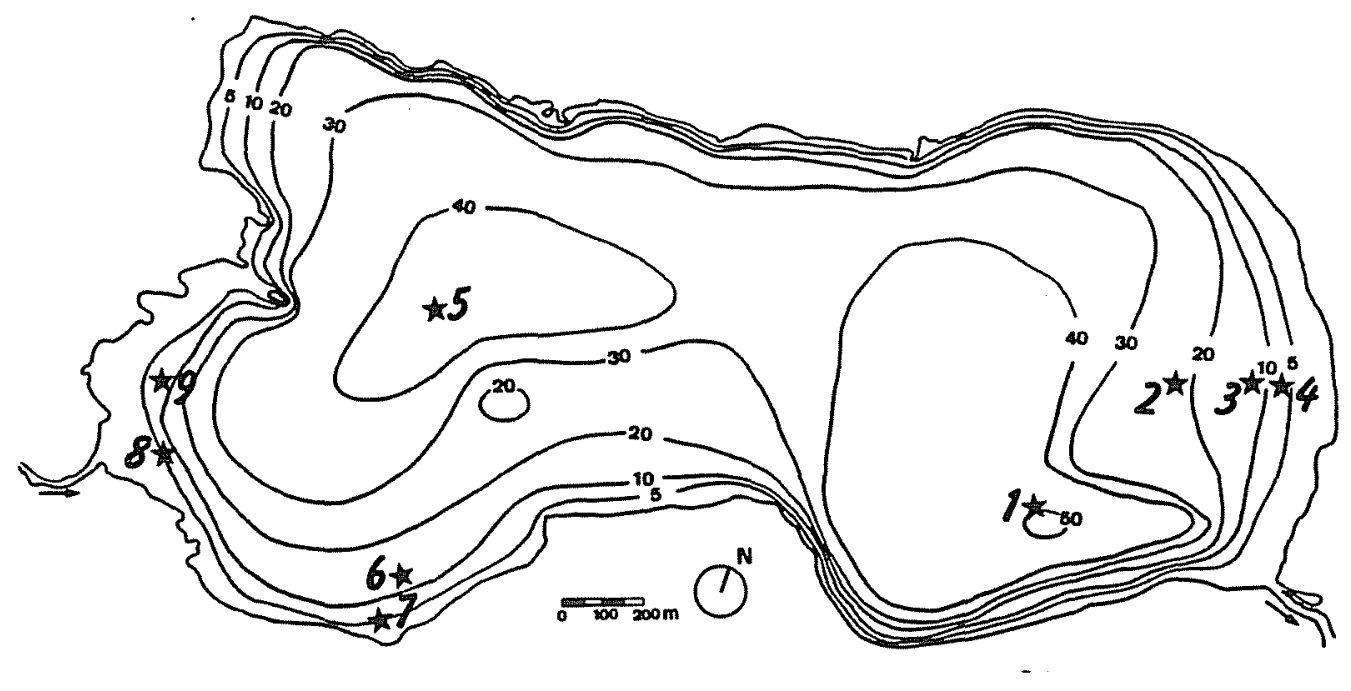

Figura 1. Mapa batimétrico del lago de Sanabria realizado por J.C. Vega (no publicado) en el que se muestran las estaciones de muestreo de bentos. La estación 1 coincide con el punto de muestreo principal en Vega et al. (en prensa).

\section{RESULTADOS}

\section{Granulometria del sedimento}

El sedimento del lago de Sanabria es de textura arcillo-limosa en las zonas profundas por debajo de 20 metros (Fig.2a) cambiando hasta texturas más gruesas que llegan a ser arenosas en las zonas sublitorales superiores muestreadas (7m) (Fig.2a). En las profundidades intermedias se encuentran mezclados materiales finos y groseros. En general puede decirse que a partir de los 12-13,5 metros (dependiendo de las zonas) se realiza el cambio de textura. El estudio detallado de la granulometría cada 2 $\mathrm{cm}$ en un gradiente vertical de un core recogido a $46 \mathrm{~m}$ de profundidad (estación 1), muestra que no hay variaciones relevantes en los primeros $14 \mathrm{~cm}$ de sedimento (Fig.2b) aumentando la fracción arenosa hacia la parte inferior del core, sin llegar a las proporciones que se dan en la zona sublitoral poco profunda donde la fracción arenosa es de casi el $90 \%$ (Fig.2a).

Tabla I. Densidad media anual de los principales grupos de macrobentos en 8 estaciones del lago de Sanabria.

$\begin{array}{lrrrrrrrr}\text { ESTACION } & 1 & 5 & 2 & 3 & 6 & 4 & 7 & 8 \\ \text { Profundidad (m) } & 46 & 40 & 22 & 13,5 & 12,5 & 8,5 & 7 & 7 \\ & & & & & & & & \\ \text { Macrobentos (ind/m²) } & & & & & & & & \\ \text { Oligoquetos } & 1833 & 4889 & 474 & 1214 & 1103 & 615 & 344 & 143 \\ \text { Quironomidos } & 518 & 859 & 333 & 767 & 1070 & 1385 & 3535 & 3541 \\ \text { Efemerópteros } & 0 & 0 & 0 & 8 & 0 & 78 & 63 & 50 \\ \text { Pisidium spp. } & 36 & 22 & 48 & 222 & 211 & 11 & 8 & 6 \\ \text { Acaros } & 4 & 4 & 15 & 107 & 41 & 103 & 19 & 15 \\ \text { Otros grupos } & 0 & 0 & 26 & 8 & 8 & 30 & 0 & 26 \\ \text { TOTAL } & 2391 & 5774 & 896 & 2326 & 2433 & 2222 & 3969 & 3781\end{array}$



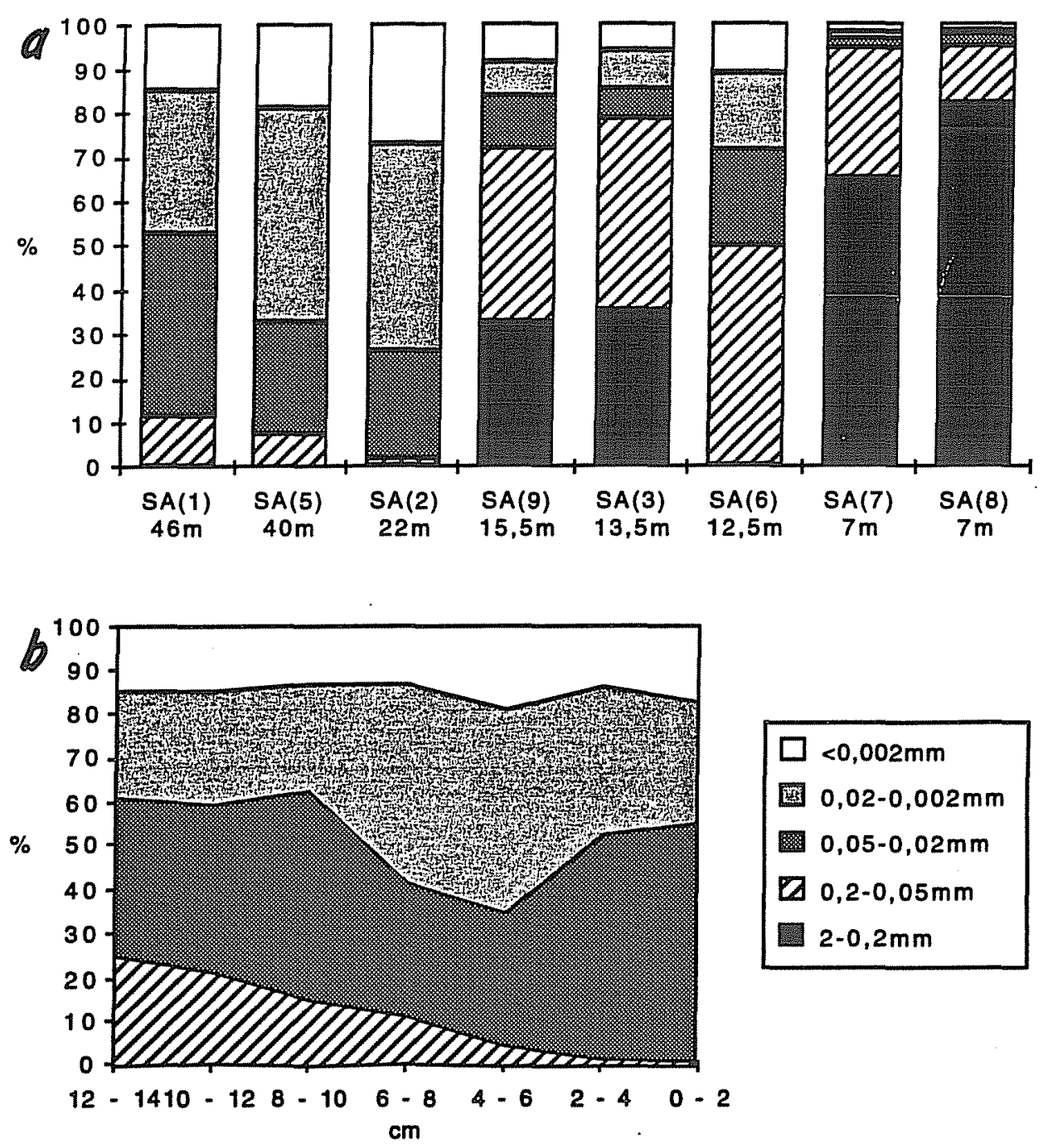

$\square<0,002 \mathrm{~mm}$
国 $0,02-0,002 \mathrm{~mm}$
网 $0,05-0,02 \mathrm{~mm}$
$\square 0,2-0,05 \mathrm{~mm}$
$\square-0,2 \mathrm{~mm}$

Figura 2. a. Datos granulométricos (en porcentages de las distintas fracciones de tamaño) en las estaciones muestreadas del lago de Sanabria; se indica la profundidad de cada estación. b. Distribución a la largo de un core de los distintos tamaños de grano del sedimento a $46 \mathrm{~m}$ de profundidad.

\section{Densidad del macrobentos y abundancia relativa de los grupos más importantes.}

Las densidades medias anuales fluctúan entre 896 ind $/ \mathrm{m}^{2}$ en la estación 2 , a $22 \mathrm{~m}$ de profundidad, hasta $5774 \mathrm{ind} / \mathrm{m}^{2}$ en la estación 5 , a 40 metros (Tabla I). No existe una correlación clara entre ninguna fracción de la granulometría y la densidad total de organismos (Tabla II).

Sí, en cambio, se obtiene una mayor correlación entre algunas fracciones granulométricas del sedimento y la relativa abundancia de los dos grupos principales, 
los quironómidos y los oligoquetos. Estos últimos son más abundantes en las zonas profundas con sedimentos finos (arcilla o limo) mientras que los quironómidos dominan en las zonas sublitorales donde la granulometría es más gruesa (con mayor importancia de la arena) (Tabla II).

En la zona profunda (más de 40 metros) la comunidad se compone casi exclusivamente de quironómidos y oligoquetos (Tabla I), siendo estos últimos el grupo dominante, con algún que otro molusco, mientras que en las zonas sublitorales, aunque los quironómidos son el grupo más abundante, existe una mayor diversidad de formas, especialmente en zonas de granulometría grosera, como los efemerópteros del género Ephemera que asimismo se correlacionan con los quironómidos.

Tabla II. Correlaciones entre las densidades medias anuales de los principales grupos de macroinvertebrados del Lago de Sanabria con las cinco fracciones granulométricas estudiadas en cada estación. Tot= total macroinvertebrados; olig= oligoquetos; quir $=$ quironómidos; efem= efemerópteros; Pisid= Pisidium, ac= ácaros; otr=otros grupos; $A G=$ arena gruesa; $A F=$ arena fina; $L G=$ limo grueso; $L F=$ limo fino; $A=$ arcilla; $Z=$ profundidad.

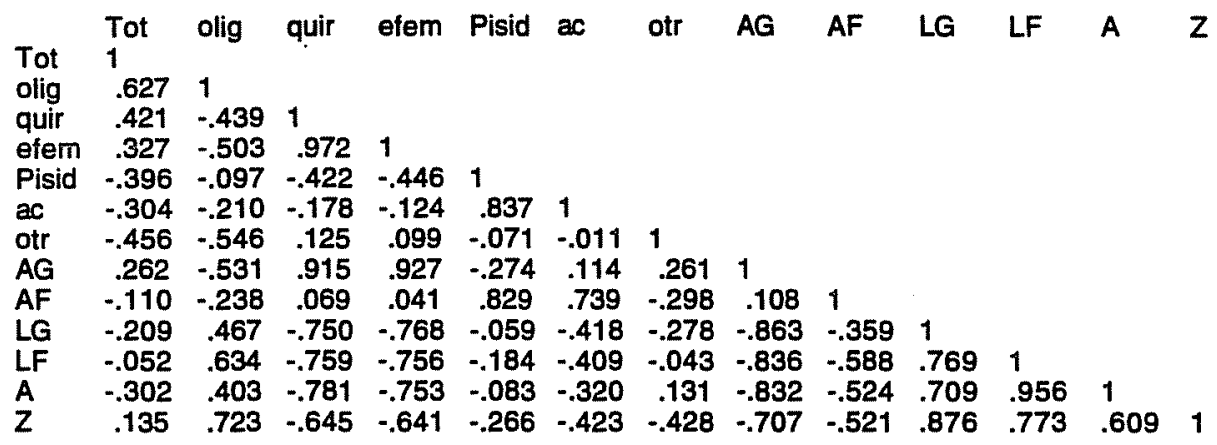

En un estudio anterior (Prat, 1980b) citamos que el quironómido de la zona profunda dominante en Sanabria era Micropsectra contracta, la misma especie que domina el bentos de lagos oligotróficos de centro Europa como el de Constanza (Reiss, 1968) o el de Stanberger (Gerstmeier, 1989). En las dragas tomadas en esta ocasión en las zonas profundas (22 metros o más) los quironómidos que se han hallado son casi exclusivamente de esta especie. Gran cantidad de los característicos tubos que construyen las larvas de este género (hechos de detritus amalgamados con la seda que ellos fabrican) tapizaban la parte superior del fango.

Otros grupos se localizan en zonas intermedias, como los bivalvos del género Pisidium o los hidrácaros (Tabla I), por lo que no es de extrañar la correlación que se da entre ellos y con las fracciones granulométricas intermedias (Tabla II).

\section{DISCUSION}

El bentos del lago de Sanabria está formado por los grupos habituales en este tipo de ambientes con dominancia de los quironómidos y oligoquetos, estos últimos son más abundantes en las zonas más profundas con sedimentos más finos tal como ocurre en muchos otros lagos y embalses. Asi ocurría en Banyoles (Rieradevall, 1991) o en los embalses españoles (Prat, 1992). La granulometría se muestra pues como un factor muy importante ya que determina en gran parte cuales son los organismos mayoritarios en el bentos del lago.

Las densidades totales son similares a las que se pueden encontrar en el lago de Banyoles o en algunos embalses poco eutróficos. Comparando con Banyoles, la zona profunda de Sanabria tiene más oligoquetos y quironómidos, mientras que en la zona sublitoral el lago de Banyoles tiene densidades más elevadas de quironómidos (ver Rieradevall, 1991; y Tabla I en Prat 1992 ). En el lago de Sanabria, el oxígeno es 
siempre abundante en todo el perfil de lago como han mostrado diferentes estudios (Planas, 1991; Vega et al., en prensa.). Este factor, pues, no debe ser clave a la hora de determinar la fauna presente en el fondo como lo era en el lago de Banyoles (Rieradevall, 1991).

En conjunto, pues, el lago de Sanabria presenta unas características muy similares a las de los lagos oligotróficos del centro de Europa (Reiss, 1968) con los que se puede comparar tanto por la presencia y abundancia de ciertas especies (Micropsectra contracta) como por las densidades recolectadas. Su similaridad a aguas de montaña y a otras lagunas españolas habia sido ya destacada anteriormente (Aldasoro et al., 1984).

El estudio detallado de las especies que colonizan el fondo del lago se realizará posteriormente. Nuestros primeros resultados coinciden con los datos de la limnología general del lago en indicar el estado oligotrófico del lago y su caráter único en España. Su conservación como Parque Natural ha sido y debe seguir siendo un factor positivo para mantener su estado actual.

\section{AGRADECIMIENTOS}

En el muestreo y separación de los macroinvertebrados han intervenido también Jordi Enériz y Manel Bolivar a los que queremos agradecer su trabajo. Muchas gracias también a José Carlos Vega que nos cedió amablemente el laboratorio del Parque Natural para trabajar así como por proporcionamos el mapa batimétrico del lago todavía, no publicado, un excelente clima de trabajo y cuantos datos adicionales le solicitamos. Asímismo el director y personal del parque Natural de Sanabria nos ha proporcionado ayuda en cuantas ocasiones la hemos requerido. Este trabajo ha contado con una, subvención de la CAYCIT, proyecto PR88-0187.

\section{BIBLIOGRAFIA}

Aldasoro, J.; C. de Hoyos; J.C. Vega \& B.G. Bikuña. 1984. Comunidades de plantas macrófitas y de crustáceos de las lagunas temporales de montaña del NW de España. Limnetica, 1: 111-115.

Anderson, R.O. 1959. A modified flotation technique for sorting bottom fauna samples. Limnol. Oceanogr. 4: 223-225.

Gerstmeier, R. 1989. Seasonal patterns in the abundance, size and production of produndal Chironomidae in Stanberger See (Bavaria, FRG). Spixiana, 12:3: 261-273.

Limnos S.A. 1989. Estudio diagnóstico de la calidad de las aguas embalsadas de la comunidad de Castilla y León: Determinación y previsión de impactos, propuesta de corrección y red de vigilancia. 3 tomos.

Martínez-Ansemil, E. \& N. Prat. 1984. Oligochaeta from profundal zones of Spanish reservoirs. Hydrobiologia, 115: 223-230.

Planas D. 1991. Factors controlling phytoplankton.community structure in an alkaline versus a softwater lake. Oecol, aquat., 10: 95-112

Prat, N. 1976. Fauna bentónica de los embalses españoles. Resúmenes II Asamblea Nacional de Geodesia y Geofísica. págs. 2041-2054.

Prat, N. 1978. Benthic typology of Spanish reservoirs. Verh. Internat. Verein. Limnol., 20: 1647-1651.

Prat, N. 1979a. Quironómidos de los embalses españoles (I). Graellsią, XXXIII: 37 96.

Prat, N. 1979b. Fauna marginal de los embalses españoles. Misc. Zoologica, 5: 149160.

Prat, N. 1980a. Quironómidos de los embalses españoles (II). Graellsia, XXXIV: 59-119.

Prat, N. 1980b. Bentos de los embalses españoles. Qecol. aquat., 4: 3-43. 
Prat, N. 1980c. Benthic population dynamics in artificial samplers in an Spanish reservoir. In: Chironomidae: Ecology. Systematics and Physiology (D.A. Murray ed.). págs, 239-246. Pergamon Press. Oxford and New York.

Prat, N. 1992. El bentos de los lagos y embalses españoles. Actas del $6^{\circ}$ Congreso Español de Limnología.

Prat, N. \& M. Rieradevall, en prensa. Life cycle and production of Cladopelma virescens (Mg.) (Dipt. Chironomidae) in lake of Banyoles (NE Spain). Neth. J. Aquat. Ecol.,26 (24).

Prat, N., J. Sanz \& E. Martínez-Ansemil, 1991. El bentos litoral y profundo de una cadena de tres embalses españoles del rio Guadiana. Limnetica, 7: 133-152.

Real, M. \& N. Prat, N. 1991. Changes in the benthos of five Spanish reservoirs in the last 15 years. Verh. Intemat. Verein. Limnol., 24: 1377-1381.

Real, M.; F. Sabater \& J.A. Morguí, 1992. Significant physiographic disturbances in the Ebro basin reservoirs (NE Spain) reflected by Oligochaeta size spectra. Hydrobiologia, 235/236: 363-374.

Reiss, F. 1968. Okologische und systematische untersuchungen aus Chironomiden (Diptera) des Bodensees. Arch. Hydrobiol., 64: 176-323.

Rieradevall, M. 1991 Ecologia i producció del bentos del llac de Banvoles. Tesis Doctoral, Univ. de Barcelona. 223 pàgs.

Rieradevall, M. \& N. Prat, 1989. Chironomidae from profundal samples of Banyoles lake. Acta Biol. Debr. Oecol. Hung., 3: 267-274.

Rieradevall, M. \& N. Prat, 1991. Benthic fauna of Banyoles lake. Verh. Intemat. Verein. Limnol., 24: 1020-1023.

Vega, J.C.; C. de Hoyos \& J. Aldosoro, en prensa. Limnologia del Lago de Sanabria. Publicaciones de la Consejeria de Medio Ambiente de la Junta de Castilla y León. 